\title{
Unable to Draw Blood
}

National Cancer Institute

\section{Source}

National Cancer Institute. Unable to Draw Blood. NCI Thesaurus. Code C113724.

Indicates that a phlebotomist was unable to find or penetrate a blood vessel or was

unable to acquire a blood sample following a successful vein stick. 